\title{
ACCURACY OF A qSOFA BASED SEPSIS SCREENING TOOL
}

Introduction: Early recognition of sepsis is vital to initiate timely treatment. The quick Sequential [Sepsisrelated] Organ Failure Assessment (qSOFA) has been proposed to identify sepsis outside the critical care setting. However there is limited evidence regarding its accuracy in the acute medical-surgical context.

Objectives: The aim of this study was to test the diagnostic accuracy of a sepsis-screening tool that incorporated qSOFA (respiratory rate $\geq 22$, altered mentation and systolic blood pressure $\leq 90 \mathrm{mmHg}$ ) and the presence of confirmed/suspected infection in general hospitalised patients. Methods: An interrupted time series study was conducted in 2017 in a private hospital in Buenos Aires. Screening was positive where the qSOFA was $\geq 2$ in patients with a confirmed or suspected infection. Screening tool performance was compared to the discharge diagnosis assessed by an experienced intensivist blinded to screening tool performance. Comorbidities were assessed with the Charlson Comorbidity Index $(\mathrm{CCl})$. Descriptive statistics and accuracy tests were conducted with SPSS ${ }^{\circledR}$ Version 25. Results: 434 patients with a median (IQR) age 70 (31) years were included. The majority were female $(n=239 ; 55.0 \%) ; 272(62.7 \%)$ were medical patients with a median (IQR) CCl 2(3). Patients stayed a median (IQR) of 4(3) days in hospital, $38(8.8 \%)$ required intensive care for 1.4 (2 days); 12 (3\%) died. The most common sources of confirmed/suspected infection were pulmonary $(79,18.2 \%)$, skin/soft tissue $(44,10.1 \%)$ and urinary $(42,9.7 \%)$. Eighty-two patients $(18.9 \%)$ had a qSOFA $\geq 2 ; 58(13.4 \%)$ were screened positive. Diagnosis at discharge were sepsis $(15,3.5 \%)$, infection $(153,35.3 \%)$ and other $(260,59.9 \%)$. Accuracy tests of the screening tool resulted in $60.0 \%$ sensitivity, $88.9 \%$ specificity, $16.4 \%$ positive and $98.4 \%$ negative predictive values. Conclusion(s): While the screening tool performed moderately well ruling-out non-septic patients, sensitivity was modest, with poor performance in predicting sepsis. 